\title{
3,4-DHPEA-EA from Olea Europaea L. is effective against standard and clinical isolates of Staphylococcus $s p$.
}

\author{
Carlo Bisignano ${ }^{1}$, Angela Filocamo², Giovanna Ginestra², Salvatore V Giofre'2, Michele Navarra², \\ Roberto Romeo ${ }^{2}$ and Giuseppina Mandalari ${ }^{*}$
}

\begin{abstract}
Background: The aim of the present work was to evaluate the antibacterial effect of 3,4-DHPEA-EA (methyl-4-(2-(3,4-dihydroxyphenethoxy)-2-oxoethyl)-3-formyl-2-methyl-3,4-dihydro-2H-pyran-5-carboxylate), a derivate of oleuropein, against a range of Gram-positive bacteria, including ATCC strains, food and clinical isolates.

Methods: The minimum inhibitory concentrations (MICS) of 3,4-DHPEA-EA were determined by the broth microdilution method and the Bioscreen C.

Results: 3,4-DHPEA-EA was effective against ATCC and clinical isolates of Staphylococcus aureus (MIC values between 125 and $250 \mu \mathrm{g} / \mathrm{ml}$ ) and ATCC and clinical isolates of Staphylococcus epidermidis (MIC values between 7.81 and $62.5 \mu \mathrm{g} / \mathrm{ml}$ ). No significant differences were observed between the two solvents (methanol and DMSO) used to dissolve 3,4-DHPEA-EA.
\end{abstract}

Conclusions: The results obtained could be used to develop novel therapies for the treatment of skin infections. Further studies need to be performed to elucidate the formation of 3,4-DHPEA-EA by acid hydrolysis of oleuropein in the human stomach.

Keywords: Olea europaea L, 3,4-DHPEA-EA, Antimicrobial, Staphylococci

\section{Background}

The potential beneficial effects of biophenols from olives (Olea europaea L.) has been observed in several studies, with antioxidant, anti-inflammatory and antimicrobial activities attributed to olive oil [1-3]. Although the health effects of olive oil were traditionally attributed to oleic acid, more recent knowledge has shown that the phenolic fraction plays a crucial role in the reported benefits [4]. Polyphenols that reach the large bowel can beneficially modulate the gut microbial ecosystem increasing the number of Bifidobacterium spp., Lactobacillus spp. and Enterococcus spp. which are known for their antiinflammatory, immunoregulatory and cholesterol lowering properties through production of short chain fatty acids $[5,6]$. The most biologically relevant compounds

\footnotetext{
* Correspondence: gmandalari@unime.it

${ }^{2}$ Dipartimento di Scienze del Farmaco e Prodotti per la Salute, University of Messina, Viale Annunziata, 98100 Messina, Italy

Full list of author information is available at the end of the article
}

contributing to the sensory and nutritional aspects of olives and olive oil are oleuropein, hydroxytyrosol, quercetin, ferulic acid, caffeic acid, $p$-hydroxybenzoic acid, protocatechuic acid, 3,4-dyhydroxyphenylacetic acid (3,4-DHPA), homovanillic acid and vanilethanediol, whose metabolic and transcriptional profiling has been recently evaluated during fruit development [7]. Oleuropein, present in large quantities in olive tree leaves and in low amounts in extravirgin olive oil, is known to be responsible for the bitter taste of the oil. We have previously demonstrated that complexes of olive biophenols with $\beta$-cyclodextrin were effective decreasing the oil bitterness and preserving from decomposition during storage [8]. The acid-hydrolysis of oleuropein in the stomach generates the formation of a number of metabolites whose distribution and concentration is dependent on the acidity of the gastric compartment [9]. The dyaldehydes originated by the cleavage of the $\beta$-glycosidic bond are unstable in the lipid/ water interface and are converted into the metabolite 
known as transposed secoiridoid or dihydropyranic form (3,4-DHPEA-EA) [10].

We have previously demonstrated that aliphatic aldehydes from Olea europeaea L. were active against human intestinal and respiratory tract infection strains, whereas Mycoplasma spp. were sensitive to oleuropein $[11,12]$. Other studies have also reported an antibacterial and antifungal action of both olive leaves and olive glutaraldehyde-like compounds $[13,14]$. A range of microbial gastrointestinal pathogens, including Escherichia coli and Helicobacter pylori, and viruses such as parainfluenza type 3 virus were sensitive to olive oil phenolic compounds [15-17].

The aim of the present work was to investigate the effectiveness of 3,4-DHPEA-EA against a range of Grampositive bacteria which included ATCC strains and food and clinical isolates.

\section{Materials and methods 3,4-DHPEA-EA}

3,4-DHPEA-EA (methyl-4-(2-(3,4-dihydroxyphenethoxy)2-oxoethyl)-3-formyl-2-methyl-3,4-dihydro-2H-pyran-5carboxylate) was obtained by enzymatic hydrolysis of oleuropein, as a molecular evolution consequence of the hemiacetal functionality of the aglycon 2 , formed by glycosidic bond cleavage (Figure 1). The lipidic/water interface promotes the rapid rearrangement of the intermediate oleuropeinenol $\mathbf{3}$ into the final stable biomolecule, the transposed secoiridoid 4, within 5 min [18].

Thus, to a solution of $100 \mathrm{mg}$ of oleuropein, previously extracted from olive leaves, endogenous $\beta$-glucosidase was added at $40^{\circ} \mathrm{C}$ for $6 \mathrm{~h}$ in $20 \mathrm{ml}$ of a $\mathrm{H}_{2} \mathrm{O} / \mathrm{CHCl}_{3} 1: 1$ mixture, The mixture was evaporated and purified using Waters XTerra $\mathrm{C}_{18}$ column on a Varian HPLC system $\left(\mathrm{H}_{2} \mathrm{O} / \mathrm{MeCN} \mathrm{H}_{2} \mathrm{O} / \mathrm{MeCN}\right.$ gradient) with a flow rate of $2 \mathrm{ml} / \mathrm{min}$.

Methyl-4-(2-(3,4-dihydroxyphenethoxy)-2-oxoethyl)-3formyl-2-methyl-3,4-dihydro-2H-pyran -5-carboxylate was obtained as a yellow oil (20\% yield). ${ }^{1} \mathrm{H}$ and ${ }^{13} \mathrm{C}$ NMR spectra were in agreement with literature data [10].

\section{Microbial strains and culture conditions}

The following Gram-positive strains were used for the antimicrobial testing and were obtained from the University of Messina's in-house culture collection (Messina, Italy):
Staphylococcus aureus ATCC 51153, Staphylococcus aureus ATCC 6538P, Staphylococcus aureus ATCC 43300, Staphylococcus epidermidis ATCC 49134, Staphylococcus epidermidis ATCC 35984, Staphylococcus epidermidis ATCC 12228, Streptococcus pneumoniae ATCC 6003, Streptococcus pyogenes ATCC 19615, Streptococcus pyogenes ATCC 10782, Listeria monocytogenes ATCC 7644, Listeria monocytogenes ATCC 1392, Enterococcus hirae ATCC 10541, Moraxella catarrhalis ATCC 8176, 10 food isolates of $L$. monocytogenes belonging to serotypes 1/2a (7 strains) and 1/2b (3 strains), 14 clinical isolates of $S$. aureus obtained from specimens of skin infections and surgical infections, 13 clinical isolates of S. pneumoniae obtained from hospitalized patients, 14 clinical isolates of $S$. pyogenes obtained from hospitalized patients, 16 clinical isolates of $M$. catarrhalis obtained from ocular and respiratory tract infections, 13 clinical isolates from $S$. epidermidis obtained from orthopedic protesis, 13 isolates of E. fecium and 15 isolates from E. faecalis from urinary tract infections.

Cultures for antimicrobial activity tests were grown either in Mueller Hinton Broth (MHB, Oxoid, CM0405, S. aureus, S. epidermidis, L. monocytogenes, E. hirae, E. fecium, E. faecalis) or Brain Heart Infusion (BHI, Difco) at $37^{\circ} \mathrm{C}(24 \mathrm{~h})$. For solid media $1.5 \%(\mathrm{w} / \mathrm{v})$ agar (Difco) was added.

\section{Antimicrobial testing}

The minimum inhibitory concentrations (MICs) of 3,4DHPEA-EA solubilized in either methanol or dimethyl sulfoxide (DMSO) were determined by the broth microdilution method, according to CLSI [19]. The MICs were also performed in the Bioscreen C (Labsystems Oy, Helsinki, Finland) for all strains as previously reported [20].

All experiments were performed in triplicate on three independent days. A number of positive and negative controls with selected antibiotics (ampicillin and ciprofloxacin) and solvents (methanol, DMSO) were included in each assay.

\section{Results}

\section{Minimum inhibitory concentrations}

The MIC values of 3,4-DHPEA-EA against the ATCC strains tested are shown in Table 1 . Results of negative controls indicated the complete absence of inhibition of

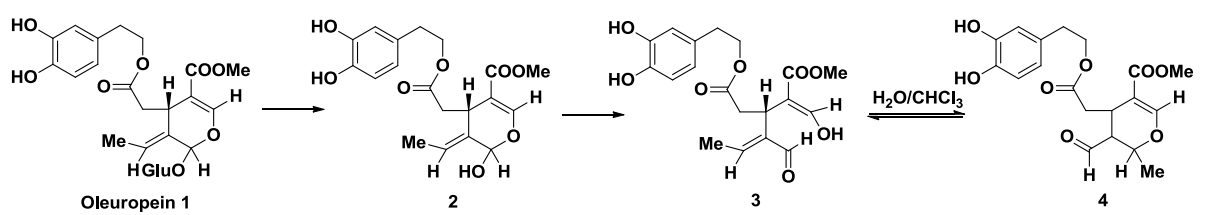

Figure 1 Synthesis of 3,4-DHPEA-EA from oleuropein. 
Table 1 Minimum inhibitory concentration (MIC) of 3,4-DHPEA-EA against ATCC Gram-positive bacteria

\begin{tabular}{lc}
\hline Strain & 3,4-DHPEA-EA \\
\hline S. aureus ATCC 51153 & 125 \\
S. aureus ATCC 6538P & 125 \\
S. aureus ATCC 43300 & 250 \\
S. epidermidis ATCC 49134 & 7.81 \\
S. epidermidis ATCC 35984 & 62.5 \\
S. epidermidis ATCC 12228 & 15.6 \\
S. pneumoniae ATCC 6003 & $>1000$ \\
S. pyogenes ATCC 19615 & $>1000$ \\
L. monocytogenes ATCC 7644 & $>1000$ \\
L. monocytogenes ATCC 1392 & $>1000$ \\
E. hirae ATCC 10541 & $>1000$ \\
M. catarrhalis ATCC 8176 & $>1000$ \\
\hline
\end{tabular}

Values are expressed as $\mu \mathrm{g} \mathrm{ml}^{-1}$ and represent the mean of three determinations. 3,4-DHPEA-EA: (methyl-4-(2-(3,4-dihydroxyphenethoxy)-2-oxoethyl)-3-formyl-2methyl-3,4-dihydro-2H-pyran-5-carboxylate).

all the strains tested (data not shown). Analogue values of MICs were obtained with the broth microdilution method and in the Bioscreen C. No differences in the MIC values were recorded with the two solvents utilized (methanol or DMSO). Amongst the Gram-positive bacteria tested, 3,4-DHPEA-EA was active against staphylococci, the most sensitive strains being $S$. epidermidis ATCC 49134 and S. epidermidis ATCC 12228, followed by $S$. aureus spp. The effect was bacteriostatic rather than bactericidal.

Table 2 reports the MICs of 3,4-DHPEA-EA against the clinical and food isolates tested. MIC values of 7.8 and $15.6 \mu \mathrm{g} \mathrm{ml}^{-1}$ 3,4-DHPEA-EA, respectively, inhibited the growth of $50 \%$ and $90 \%$ of the $S$. epidermidis strains tested, whereas 125 and $250 \mu \mathrm{g} \mathrm{ml}^{-1}$ 3,4-DHPEA-EA, respectively, inhibited the growth of $50 \%$ and $90 \%$ of the $S$. aureus strains tested. All the other isolates were

Table 2 Minimum inhibitory concentration (MIC) of 3,4-DHPEA-EA against food and clinical isolates

\begin{tabular}{lcc}
\hline Strain & MIC 50 & MIC 90 \\
\hline S. aureus & 125 & 250 \\
S. epidermidis & 7.8 & 15.6 \\
S. pyogenes & $>1000$ & $>1000$ \\
S. pneumoniae & $>1000$ & $>1000$ \\
M. catarrhalis & $>1000$ & $>1000$ \\
L. monocytogenes & $>1000$ & $>1000$ \\
E. faecalis & $>1000$ & $>1000$ \\
E. fecium & $>1000$ & $>1000$ \\
\hline
\end{tabular}

Values are expressed as $\mu \mathrm{g} \mathrm{ml}^{-1}$ and represent the mean of three determinations. 3,4-DHPEA-EA: (methyl-4-(2-(3,4-dihydroxyphenethoxy)-2-oxoethyl)-3-formyl-2methyl-3,4-dihydro-2H-pyran-5-carboxylate). resistant. Higher MIC values were obtained with S. aureus compared to S. epidermidis (Table 2).

\section{Discussion}

The present study has evaluated the antimicrobial effect of a metabolite from oleuropein, 3,4-DHPEA-EA, against a range of Gram-positive bacteria, which included ATCC strains, food and clinical isolates. We have recently demonstrated that polyphenols from pistachios had a bactericidal effect against $S$. aureus and L. monocytogenes [21], whereas almond skin extracts rich in polyphenols were active against a range of Gram-positive bacteria [22]. The effect on $S$. aureus could be used to find potential applications as a topical treatment for $S$. aureus. Other authors have reported on the antioxidant and antimicrobial activities of individual and combined phenolics in Olea europea leaf extract: oleuropein and caffeic acid were active against Salmonella enteridis, Bacillus cereus and Escherichia coli and the antimicrobial effect of the combined phenolics was significantly higher than those of the individual compounds [23]. Using agar dilution and broth microdilution techniques, Sudjana et al. [24] found that a commercial olive leaf extract was active against Campylobacter jejuni, Helicobacter pylori and Staphylococcus aureus with low MIC concentrations $(0.31-0.78 \% \mathrm{v} / \mathrm{v})$. Another investigation on the antimicrobial effect of an olive leaf extract showed that Bacillus subtilis was less susceptible than E. coli, Pseudomonas aeruginosa, Klebsiella pneumoniae and S. aureus [25]. A commercial olive powder and 4-hydroxytyrosol were able to inactivate $S$. aureus and its enterotoxin A, secreted by the bacteria in $78 \%$ of the outbreaks [26]. Although no reports have identified the possible mechanisms of action of the phenolic compounds present in olive leaf, some authors report the activity of phenolics on Gram-positive bacteria may be due to the cell wall or cell membrane disruption together with cell enlargement, which is more susceptible compared to Gram-negative strains [27]. Fabiani et al. [28] demonstrated that hydrogen peroxidase production is responsible for the induction of apoptosis by hydroxytyrosol on HL60 cells.

In our previous study we demonstrated that oleuropein and hydroxytyrosol were active against ATCC strains and clinical isolates: the MIC values of hydroxy-

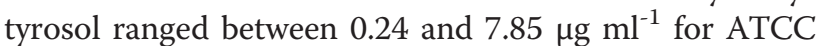
strains and between 0.97 and $31.25 \mu \mathrm{g} \mathrm{ml}^{-1}$ for clinically isolated strains, whereas the MIC values of oleuropein ranged between 62 and $500 \mu \mathrm{g} \mathrm{ml}^{-1}$ for ATCC strains and between 31.25 and $250 \mu \mathrm{g} \mathrm{ml}^{-1}$ for clinically isolated strains [3]. Although oleuropein was found effective against a range of Gram-positive strains, in the present work we demonstrated that 3,4-DHPEA-EA, a metabolite obtained by hydrolysis of oleuropein, was only active against ATCC and clinical isolates of S. aureus and 
S. epidermidis. The use of Olea metabolites could therefore be tested in combination with traditional antibiotics in order to identify new mechanisms of synergism and antibiotic-resistant modulating properties for the development of novel drugs. On the basis of previous investigations on the absorption and metabolism of olive oil secoiridoids in the small intestine [29], further studies need to be performed to elucidate the formation of 3,4DHPEA-EA in the stomach.

\section{Conclusions}

In summary, the results of the present study showed that a metabolite from oleuropein was effective against staphylococci and could therefore be a potential source of natural antimicrobials for the treatment of skin infections. However, further studies are needed to understand the mechanisms responsible for these activities and the obtained in vitro results need to be translated both in food and in vivo.

\section{Competing interest}

The authors declare no conflict of interest.

\section{Authors' contributions}

GM designed research, CB, AF, GG, SVG carried out research, GM, MN, RR analysed results, GM wrote the manuscript. All authors read and approved the final manuscript.

\section{Acknowledgements}

This research was funded by the University of Messina and INBB (Consorzio interuniversitario Istituto Nazionale di Biostrutture e Biosistemi).

\section{Author details}

Dipartimento di Scienze Biologiche ed Ambientali, University of Messina, Salita Sperone 31, 98100 Messina, Italy. ${ }^{2}$ Dipartimento di Scienze del Farmaco e Prodotti per la Salute, University of Messina, Viale Annunziata, 98100 Messina, Italy.

Received: 4 February 2014 Accepted: 27 May 2014 Published: 1 July 2014

\section{References}

1. Martin-Pelaez S, Covas MI, Fito M, Kusar A, Pravst I: Health effects of olive oil polyphenols: recent advances and possibilities for the use of health claims. Mol Nutr Food Res 2010, 57:760-771.

2. Cicerale S, Conlan XA, Sinclair AJ, Keast RSJ: Chemistry and health of olive oil phenolics. Crit Rev Food Sci Nutr 2009, 49:218-246.

3. Bisignano G, Tomaino A, Lo Cascio R, Crisafi G, Uccella N, Saija A: On the in-vitro antimicrobial activity of oleuropein and hydroxytyrosol. J Pharm Pharmacol 1999, 51:971-974.

4. Omar SH: Oleuropein in olive and its pharmacological effects. Sci Pharm 2010, 78:133-154.

5. Martinez I, Wallace G, Zhang CM, Legge R, Benson AK, Carr TP, Moriyama EN, Walter J: Diet-induced metabolic improvements in a hamster model of hypercholesterolemia are strongly linked to alterations of the gut microbiota. Appl Environ Microbiol 2009, 75:4175-4184.

6. Trautwein EA, Rieckhoff D, Erbersdobler HF: Dietary inulin lowers plasma cholesterol and triacylglycerol and alters biliary bile acid profile in hamster. J Nutr 1998, 128:1937-1943.

7. Alagna F, Mariotti R, Panara F, Caporali S, Urbani S, Veneziani G, Esposto S, Taticchi A, Rosati A, Rao R, Perrotta G, Servili M, Baldoni L: Olive phenolic compounds: metabolic and trabscriptional profiling during fruit development. BMC Plant Biol 2012, 12:162-180.

8. Rescifina A, Chiacchio U, lannazzo D, Piperno A, Romeo G: $\beta$-cyclodextrin and caffeine complexes with natural polyphenols from olive and olive oils: NMR, thermodynamic, and molecular modeling studies. J Agric Food Chem 2010, 58:11876-11882

9. Carrera-Gonzales MP, Ramirez-Exposito MJ, Mayas MD, Martinez-Martos JM: Protective role of oleuropein and its metabolite hydroxytyrosol on cancer. Trends Food Sci Technol 2013, 31:92-99.

10. Daccache A, Lion C, Sibille N, Gerard M, Slomianny C, Lippens G, Cotelle P: Oleuropein and derivatives from olives as Tau aggregation inhibitors. Neurochem Int 2011, 58:700-707.

11. Bisignano G, Lagana' MG, Trombetta D, Arena S, Nostro A, Uccella N Mazzanti G, Saija A: In vitro antibacterial activity of some aliphatic aldehydes from Olea europaea L. FEMS Microbiol Lett 2001, 198:9-13.

12. Furneri PM, Marino A, Saija A, Uccella N, Bisignano G: In vitro antimycoplasmal activity of oleuropein. Int J Ant Ag 2002, 20:293-296.

13. Medina E, Brenes M, Garcia A, Romero C, de Castro A: Bactericidal activity of glutaraldehyde-like compounds from olive products. J Food Prot 2009, 72:2611-2614

14. Pereira AP, Ferreira IC, Marcelino F, Valentao P, Andrade PB, Seabra $R$, Estevinho L, Bento A, Pereira JA: Phenolic compounds and antimicrobial activity of olive (Olea europaea L. Cv. Cobrancosa) leaves. Molecules 2007, 12:1153-1162.

15. Medina E, Brenes M, Romero C, Garcia A, de Castro A: Main antimicrobial compounds in table olives. J Agric Food Chem 2007, 55:9817-9823.

16. Romero C, Medina E, Vargas J, Brenes M, de Castro A: In vitro activity of olive oil polyphenols against Helicobacter pylori. J Agric Food Chem 2007 55:680-686.

17. Ma SC, He ZD, Deng XL, But PPH, Ooi VE, Xu HX, Lee SH, Lee SF: In vitro evaluation of secoiridoid glucosides from the fruit of Ligustrum lucidum as antiviral agents. Chem Pharm Bull 2001, 49:1471-1473.

18. Bianco AD, Piperno A, Romeo G, Uccella N: NMR experiments of oleuropein biomimetic hydrolysis. J Agric Food Chem 1999, 47:3665-3668.

19. Clinical and Laboratory Standards Institute, M100-S18: Performance Standards for Antimicrobial Susceptibility Testing; Seventeenth Informational Supplement. Wayne, PA: Clinical Laboratory Standards Institute; 2008.

20. D’Arrigo M, Ginestra G, Mandalari G, Furneri PM, Bisignano G: Synergism and postantibiotic effect of tobramycin and Melaleuca alternifolia (tea tree) oil against Staphylococcus aureus and Escherichia coli. Phytomedicine 2010, 17:317-322

21. Bisignano C, Filocamo A, Faulks RM, Mandalari G: In vitro antimicrobial activity of pistachio (Pistacia vera L.) polyphenols. FEMS Microbiol Lett 2013, 341:62-67.

22. Mandalari G, Bisignano C, D'Arrigo M, Ginestra G, Arena A, Tomaino A, Wickham MSJ: Antimicrobial potential of polyphenols extracted from almond skins. Lett Appl Microbiol 2010, 51:83-89.

23. Lee O-H, Lee B-Y: Antioxidant and antimicrobial activities of individual and combined phenolics in Olea europaea leaf extract. Bior Technol 2010, 101:3751-3754.

24. Sudjana AN, D'Orazio C, Ryan V, Rasool N, Ng J, Islam N, Riley TV, Hammer KA: Antimicrobial activity of commercial Olea europaea (olive) leaf extract. Int J Ant Ag 2009, 33:461-463.

25. Markin D, Duek L, Berdicevsky I: In vitro antimicrobial activity of olive leaves. Mycoses 2003, 46:132-136.

26. Friedman M, Roasooly R, Do PM, Henika PR: The olive compound 4-hydroxytyrosol inactivates Staphylococcus aureus bacteria and Staphylococcical enterotoxin A (SEA). J Food Sci 2011, 76:M558-M563.

27. Al-Habib A, Al-Saleh E, Safer A-M, Afzal M: Bactericidal effect of grape seed extract on methicillin-resistant Staphylococcus aureus (MRSA). J Toxicol Sci 2010, 35:357-364

28. Fabiani R, Fuccelli R, Pieravanti F, De Bartolomeo A, Morozzi G: Production of hydrogen peroxidase for the induction of apoptosis by hydroxytyrosol on HL60 cells. Mol Nutr Food Res 2009, 53:887-896.

29. Pinto J, Paiva-Martins F, Corona G, Debnam ES, Jose Oruna-Concha M, Vauzour D, Gordon MH, Spencer JP: Absorption and metabolism of olive oil secoiridoids in the small intestine. Br J Nutr 2011, 105:1607-1618.

doi:10.1186/1476-0711-13-24

Cite this article as: Bisignano et al: 3,4-DHPEA-EA from Olea Europaea L. is effective against standard and clinical isolates of Staphylococcus sp. Annals of Clinical Microbiology and Antimicrobials 2014 13:24. 